\title{
POUR ÉCRIRE UNE HISTOIRE LITTÉRAIRE : L’EXEMPLE DE CALELHON
}

Catherine Parayre, Brock University

Le vingtième siècle a peu prisé l'histoire littéraire. Plus généralement, il a souvent remis en question le rôle de la littérature. Par exemple,

\begin{abstract}
Au moment où J. Hillis Miller, en Amérique, dénonce toute la littérature comme une imposture, Barthes, jouant sur la distinction saussurienne entre un mot et une chose, la caractérise comme un système trompeur de significations. Plus apocalyptique, Blanchot prophétise la disparition de la littérature et du même coup la mort du dernier écrivain. (Sturm 19)
\end{abstract}

Quant aux recherches portant sur I'histoire littéraire, elles n'ont pas toujours privilégié la dimension historique, sociale et politique de l'écriture (16). Dans Les Fondements de I'histoire littéraire, Luc Fraisse remarque : «Donner la parole à l'histoire littéraire constituerait à soi seul une nouveauté : on lui a longtemps, surtout durant ces dernières décennies, refusé cette parole »(7). Néanmoins, I'histoire littéraire est une discipline à part entière, qui, à ce titre, mérite l'attention. En particulier, et c'est ce qui nous intéressera ici, la situation des langues minoritaires soulève des questions qui révèlent combien il est important de produire une histoire de la littérature. En effet, dans le cas d'une langue menacée, les contraintes sociohistoriques peuvent difficilement être passées sous silence, car le problème qui se pose n'est pas uniquement celui du prestige et du rôle de la littérature, mais, avant toute chose, celui de son existence et de sa distribution. Pouvoir ignorer l'environnement social de la littérature ou estimer que la littérature n'est autre qu'un luxe intellectuel équivoque, voilà deux privilèges dont ne disposent pas certaines littératures. Les langues minoritaires sont des langues qui, pour des raisons de survie, ont besoin d'histoire et leur littérature ne peut pas être traitée comme un pur objet esthétique.

Il s'agira d'examiner l'exemple d'une histoire littéraire de l'occitan (langue minoritaire traditionnellement parlée dans le sud de la France), plus précisément un ouvrage qui est lui-même méconnu dans la littérature occitane, le Cours de littérature occitane (1944) de Calelhon (Julienne Fraysse-Séguret à l'état-civil, 1891-1981). De fait, ce sont des ouvrages ultérieurs, celui de Charles Camproux (1953) et celui de Robert Lafont et Christian Anatole (1971) qui font référence parmi les chercheurs en occitan. Celui de Calelhon est entièrement oublié de nos jours, bien qu'il discute un problème capital pour la langue occitane, celui du passage de l'oral à l'écrit en milieu scolaire. Camproux d'une part, et Lafont et Anatole d'autre part font œuvre universitaire et destinent leurs travaux à un public cultivé déjà sensibilisé à la problématique occitane. Comme le suggère la présentation du livre de Calelhon, son histoire littéraire est une introduction générale pour les élèves scolarisés en Rouergue (département de l'Aveyron). La présente étude examinera le contenu du Cours, mais aussi le parcours littéraire de l'auteure, pour conclure sur le contexte socioculturel occitan et français de ce projet. Elle montrera qu'en dépit du souci d'innovation et d'historicité qui le caractérise, l'ouvrage évite toute prise de position conflictuelle. Paradoxalement, celui-ci brouille et parfois efface tout engagement sociopolitique qui serait ouvertement oppositionnel, alors qu'il invoque la nécessité d'une analyse nouvelle des circonstances historiques qui ont joué un rôle dans l'affaiblissement de la langue. C'est ce mélange de revendication d'une 
histoire et de refus d'histoire (voir Lafont 35-36), comme si la littérature construisait I'histoire tout en I'annulant, qui donne son intérêt à I'histoire littéraire qu'a rédigée Calelhon.

Née en 1891 à Privezac, petit village aveyronnais, Calelhon grandit dans un milieu rural, où, entourée de locuteurs occitans, elle apprend les coutumes et légendes transmises dans cette langue. Elle développe déjà jeune un goût prononcé pour la littérature et, consciente des avantages socioéconomiques que procure l'éducation, entreprend des études d'institutrice. Tout en exerçant ce métier, Calelhon connaît un parcours littéraire occitan bien rempli. A la maison, elle a découvert l'œuvre de Frédéric Mistral, grand poète de langue occitane, fondateur du Félibrige, mouvement de défense de la langue, et prix Nobel de littérature (1904). De là viennent l'adhésion de la jeune femme au Félibrige, de même que son propre travail d'écriture et son engagement dans les cercles littéraires du Rouergue. Parmi ses ouvrages, on peut citer Lo Temps perdut (1967), recueil de poèmes, et Contes del Papanon (1971). En 1920, elle est co-fondatrice du « Grelh Roergàs », groupement littéraire rouergat. Avec son mari, elle mène campagne auprès des autorités locales pour que soit donné un enseignement en occitan. Tous deux finissent par convaincre la direction du Lycée de Rodez et Calelhon forme alors une équipe de quatre enseignants (Séguret 100). En 1940, soixante-quatre élèves participent aux cours et on doit refuser des inscriptions (Fourié 136). En 1941, Calelhon informe des amis qu'elle travaille à un «libre de lecturas rouergatas »(136). Toutefois, la frilosité administrative va parfois de pair avec une hostilité avouée dans certaines couches de la population. Par exemple, Le Journal de l'Aveyron du 4 mars 1944 contient un article peu encourageant :

Inutile d'enseigner la langue maternelle aux fils de paysans. Ils la parlent mieux que leurs maîtres.... Les parents envoient leurs enfants à l'école pour apprendre le français.... La meilleure façon de conserver la pureté de la langue, ce n'est pas de l'écrire, ni surtout de I'enseigner. (cité dans Séguret 101)

C'est donc dans le but de légitimer l'occitan en tant que langue d'écriture que Calelhon écrit le texte des cours qu'elle entend enseigner à Rodez. Long de soixante et onze pages, le Cours est essentiellement un cours de littérature rouergate. Certes, plusieurs chapitres sont consacrés à des sujets généraux et contiennent de nombreux commentaires sur les troubadours, le poète agenais Jasmin (1798-1864), Frédéric Mistral (18301914) et le Félibrige. Toutefois, cette histoire commune au sud de la France est souvent ramenée à ses implications en milieu rouergat et il est fait grand cas de plusieurs écrivains locaux. Le deuxième chapitre s'intitule «Un poète rouergat du XVIIIe siècle : Claude Peyrot » et les deux derniers sont «La 'respelida' en Rouergue » (c'est-à-dire la renaissance) et « Le mouvement contemporain : le Grelh Rouergat ». L'information communiquée est accompagnée d'indications bibliographiques et inclut des exercices ou des suggestions de lecture. Huit chapitres sur onze analysent la production littéraire du dix-neuvième siècle, principalement le Félibrige. L'auteur alterne histoire, histoire de la langue, histoire de la littérature, résumés et explication d'œuvres. La première page donne le ton : « Notre dialecte rouergat que nous avons le tort d'appeler un patois, n'est autre que le languedocien. La langue d'oc n'est donc pas un patois ; elle est une vraie langue » (6). Pour affirmer la continuité littéraire, Calelhon prend soin de souligner les périodes de transition entre différentes pratiques d'écriture. L'exemple le plus frappant concerne le Félibrige. Calelhon situe Jasmin comme un précurseur du mouvement fondé par Mistral et ses amis en 1854. En Rouergue, elle nomme un poète 
pratiquement inconnu, Bernard d'Armagnac, comme appartenant au «mouvement précurseur du Félibrige » (17). Bien plus, elle avance trois conditions majeures qui ont favorisé la création de l'association. La première est le «mouvement des nationalités » qu'a connu le dix-neuvième siècle : «En France, où l'unité est faite et solidement assise, l'idée nationale va prendre la forme régionale ; ce sera le mouvement de la renaissance des provinces» (16). Le deuxième motif de cette «respelida» est, selon Calelhon, le travail de recherche des « historiens et érudits » depuis le dix-neuvième siècle. S'appuyant sur La Renaissance provençale d'Emile Ripert (1918), elle fait mention de travaux savants en matière de littérature médiévale. Finalement, elle voit dans la poésie populaire des régions, dont elle situe l'apogée vers 1830, à l'époque de George Sand, Alphonse de Lamartine et Jules Michelet, un facteur de sensibilisation à la cause régionale (16-17).

Une fois présentés les événements précurseurs du Félibrige, Calelhon s'attarde sur la description de I'association. Au portrait flatteur de Mistral, dépeint comme un homme de la terre, succède une courte définition des buts du Félibrige : «Défendre, répandre, illustrer la langue d'oc et, avec elle et par elle, les traditions, les mœurs, les croyances et toutes les libertés de la province. C'était tout un programme non seulement littéraire mais aussi social, administratif et politique. 'Nous faisons, pacifiques, une révolution', pourra dire plus tard Mistral »(25). Malgré le bouleversement annoncé, Calelhon omet d'éclaircir plus profondément la politique linguistique et culturelle du Félibrige et, par ce flou, dépolitise la pensée de Mistral. En fait, dans un chapitre subséquent, elle relève dans son œuvre les seuls traits qui servent à rassembler les opinions plutôt qu'à les diviser sur des sujets conflictuels: Mistral exprime «les sentiments communs à la plupart des hommes, du moins des hommes d'un même pays ; ce qui peut les unir, les faire vivre ensemble, les faire communier dans une même foi, un même idéal »(44). Elle pense aussi de lui qu'il est un grand « poète civique » qui a « célébré les grandes gloires nationales» (44), véritable sage avec « tout un côté de poésie familière, de bonhomie populaire, de simplicité rustique » (48).

De la renaissance provençale, Calelhon passe à la renaissance languedocienne avec, en particulier, la fondation de l'Ecole Occitane et du Collège d'Occitanie à Toulouse. C'est à ces deux institutions qu'elle rattache les écrivains rouergats. Elle n'oublie pas d'inclure ses écoliers dans ce renouveau :

En Rouergue des cours de langue d'oc et de graphie occitane sont donnés au Lycée, au Collège Fabre, à I'Institution Sainte-Marie, au Grand Séminaire et dans un certain nombre de petits séminaires.... Depuis [1942], profitant du décret Carcopino, qui permet l'enseignement facultatif dialectal dans les écoles primaires, beaucoup d'instituteurs ruraux enseignent la langue d'oc et les principes de la graphie classique occitane. (61)

Dans son enthousiasme, Calelhon exagère vraisemblablement l'engouement du corps enseignant pour I'apprentissage de I'occitan en Rouergue et on ne saurait imaginer un succès fulgurant de cette langue dans les écoles aveyronnaises. La fin du Cours s'intéresse exclusivement aux écrivains rouergats : Justin Bessou pour la « respelida » en Rouergue, Henri Mouly et le couple Calelhon-Séguret pour le « mouvement contemporain : le Grelh Rouergat ». Calelhon précise l'importance d'une graphie standardisée (à cette époque, l'occitan est essentiellement langue orale): «C'est donc I'orthographie classique qui est enseignée aux élèves de nos grandes et petites écoles. Il est à remarquer que, de plus en plus, les meilleurs écrivains de toutes les provinces méridionales... adoptent I'orthographe occitane classique »(68). La question de la graphie, souvent reprise dans l'ouvrage, est débattue comme étant un point central dans la transmission de la langue. Cette 
insistance est compréhensible : I'objectif de Calelhon est d'introduire l'occitan à l'école en tant que langue littéraire. Une courte conclusion affirme que la langue des paysans rouergats est une langue poétique (70). Dans l'ensemble, le Cours confère une double autorité à Calelhon : cette dernière se présente à la fois comme pédagogue avertie (capable d'écrire un manuel scolaire) et femme de lettres reconnue (parmi ceux qu'elle estime être les meilleurs écrivains).

Les histoires littéraires de Camproux d'une part, et de Lafont et Anatole d'autre part, postérieures à celle de Calelhon, suivent une orientation différente. Ces deux ouvrages, bien plus épais que celui de Calelhon et destinés à un public tout autre, regorgent de détails et d'analyses, mais ne font pas du Félibrige l'entreprise la plus remarquable de la tradition occitane. Plus encore, Camproux tout comme Lafont et Anatole n'hésitent pas à faire part d'interprétations tranchées sur les questions traitées, ce que Calelhon ne fait pas. Certes, cette dernière réclame qu'il soit accordé un plus grand rôle aux régions, mais elle n'avance aucune position politique nettement établie. (Ses positions les plus distinctes sont réservées aux questions de la graphie et de l'existence même d'une littérature occitane). Camproux, de même que Lafont et Anatole s'engagent bien plus résolument. Camproux réfute carrément la notion d'une « nation occitane » (6) et estime que la littérature occitane trouve sa spécificité dans la langue : «Elle est la littérature d'une langue.... Fait d'autant plus original que cette langue ne fut jamais une langue 'politique'»(7). Lafont et Anatole, quant à eux, expriment sans détours une revendication politique. Ils souhaitent historiciser la littérature occitane et surtout la rendre égale aux autres littératures romanes. Contrairement à Calelhon, qui compare fréquemment les textes occitans aux textes français, Lafont et Anatole se refusent à cette pratique et étudient la production littéraire occitane en tant que production autonome.

Par exemple, les trois études reconnaissent l'œuvre du Rouergat Peyrot, mais pour des raisons dissemblables. Calelhon décrit cet auteur comme un prêtre aimable goûtant la vie des champs (13). Bien qu'elle juge « insupportable » la graphie occitane qu'il utilise, « émaillée de gallicismes, chargée d'impuretés,... trop souvent français patoisé »(13), elle apprécie la verve populaire et le pittoresque rural de l'œuvre. Pour mieux asseoir sa démonstration, elle fait appel à une critique parue dans Le Mercure du 8 juin 1782 et à une anecdote rapportant que le futur Louis XVIII avait pris plaisir à écouter la récitation des poèmes de Peyrot. Toutefois, elle précise que Peyrot est passé de mode : «Nous le goûtons moins aujourd'hui » (13). Camproux reprend les citations du Mercure et l'anecdote royale, mais se garde bien de reprocher au poète la graphie de ses écrits. Bien au contraire, il s'exclame, avec peut-être un peu trop de confiance : «Rien d'étonnant si I'œuvre virgilienne de Peyrot n'a pas vieilli et si elle est demeurée populaire en Rouergue » (125). Alors que Calelhon reproche aux écrits de Peyrot de ne plus correspondre aux normes désormais en vigueur, Camproux voit chez Peyrot un auteur dont l'œuvre ne saurait se démoder. Le travail de Lafont et Anatole contredit également celui de Calelhon. Il ne fait aucun commentaire sur la langue qu'utilise Peyrot. En revanche, il indique que celui-ci «pactisera avec la Révolution [et] célèbrera en 1793 'I'arbre de la Liberté' » (477). On est bien loin du portrait paisible du Cours de Calelhon ; Peyrot est à présent un personnage quasiment révolutionnaire. Un tel exemple est caractéristique des divergences de vues dans les trois histoires littéraires. Généralement, Calelhon évalue les œuvres qu'elle décrit selon un critère régional qui fait de la langue et de la 
ruralité deux aspects majeurs. Si le critère linguistique n'est pas respecté, son jugement est sévère, contrairement à Camproux, qui s'intéresse à une approche plus littéraire. En outre, Calelhon cherche, le plus souvent, à innocenter, en quelque sorte, la littérature. Alors que Lafont et Anatole mettent en relief les conflits et les enjeux sociaux qui alimentent le débat littéraire, Calelhon préfère dresser un tableau idyllique de I'artiste inspiré, sage et philosophe. Il reste à ajouter que Camproux place Calelhon et son mari dans la liste des auteurs de I'Ecole Occitane, sans plus s'attarder sur la nature de l'œuvre de Calelhon. Bien qu'elle comporte un chapitre sur le Rouergue et le Gévaudan, I'histoire littéraire de Lafont et Anatole ne contient aucune mention des textes de Calelhon. Il est juste d'indiquer que le Cours de Calelhon a laissé peu de traces dans la critique occitane.

Celui-ci est pourtant le fruit d'une réflexion méticuleuse de la part de son auteure, qui n'a pas abandonné le sujet à la parution de son ouvrage. En fait, le sujet est repris, parfois dans les mêmes termes, souvent d'un point de vue différent, dans Lo Pan tendre: Al païs negre, récit autobiographique de Calelhon. Deux points principaux caractérisent les souvenirs de l'auteure, leur ton polémique d'une part et le besoin d'authenticité d'autre part. Au cours de ses études, Calelhon se prend de passion pour la littérature française, mais c'est la découverte de Mirèio, chef-d'œuvre de Mistral, qui comble ses attentes. Elle est séduite par la sincérité du ton et s'identifie à l'héroïne, comme elle adolescente dans un milieu social ordonné par une hiérarchie stricte :

D'esprit e de còr me sentissiai en comunion ambe aquèla subre-bèla e tant umana Poesia. E per bona escasença, abiai la clau d'aquel trèsor : la lenga mia, la lenga nòstra, la lenga d'Oc. (217)

D'instinct je sentais tout cela. Je communiais vraiment. D'où venait cette facilité ? Comment tant de beauté pouvait-elle m'être si accessible et presque familière ? - Parce que je possédais la clé du trésor ; ma langue maternelle, la langue d’oc. (216)

Contrairement au Cours, ces pages évitent de comparer la littérature occitane à la littérature française. Pourtant, la prise de conscience de la jeune fille donne lieu à un passage crucial dans la narration. L'original occitan célèbre la seule «civilisation occitane »: «Dins la polvera de demembrança amassèri la claù d'ór, lo tresòr de la Poesia ; la civilisacion occitana m'apareguèt » (219). La traduction (Calelhon s'auto-traduit) transforme la nature de la découverte : «Dans la poussière de l'oubli, je ramassai la clé d'or et le trésor d'une double culture française et occitane me fut offert » (226). Cette traduction révèle la subtilité de l'auteure dans sa démarche. Calelhon cherche à atteindre deux types de public, les occitanophones et ceux qui ne peuvent lire que la traduction française. Elle adapte donc son message pour ce dernier public, comme en de nombreux autres moments du récit. Il s'agit sans doute de ne pas aliéner ce lecteur du français et de donner un peu de crédit à la culture française.

De plus, et contrairement au contenu du Cours, il n'y a dans le chapitre consacré à la littérature que contient le Pan tendre, aucune envolée patriotique et aucun discours sur I'unité de la nation française. (II faut dire que les mémoires de Calelhon témoignent d'une période antérieure à la première guerre mondiale). En revanche, Calelhon se fait volontiers porte-parole d'une revendication absente dans le Cours. Dans Lo Pan tendre, elle se mobilise pour «lo pòple occitan » / « le peuple occitan » et réclame « de dreches a far valer » / «l'affirmation de ses droits»(220-221). Alors que le Cours enseigne une langue et sa littérature, 
I'autobiographie défend une cause linguistique tout autant que politique, à savoir «l'Idée occitane » (222). De nouveau, la traduction éclaire la stratégie à double tranchant qu'emploie Calelhon. « L'Idée occitane », terme vague pouvant s'appliquer à divers contextes, n'apparaît que dans la traduction, mais le passage entier établit une distinction capitale entre la revendication d'une identité culturelle, géographiquement délimitée, dans I'original occitan et, dans le texte français, la défense d'un programme linguistique :

Artistas e sabents, escrivans e trobaires, totes afortissian que la lenga nostra èra sempre viventa e que lo Païs d'Oc abia drech a la vida e a la libertat. La Causa èra justa e bèla. (221)

Artistes, savants, poètes, écrivains affirmaient la vitalité de notre langue et réclamaient ses droits. La cause me parut juste et généreuse. (222)

Et voilà Calelhon qui se lance dans un vaste projet d'engagement. Toutefois, la cause change en changeant de langue : le double objectif de défense d'une langue et d'une région se réduit, dans sa traduction, à l'unique objectif de la promotion linguistique.

Or, cette modération se retrouve dans le Cours et il convient de garder en mémoire le travail d'adaptation qu'effectue Calelhon dans la traduction du Pan tendre lorsqu'on examine le Cours. Rédigé en français, celui-ci est destiné à être lu dans l'institution qui garantit la transmission du français, l'école, et on peut estimer que l'auteur ménage son propos dans un tel environnement. En fait, le livre est tout simplement confronté au défi que représente l'emploi d'un texte d'histoire à l'école. Essentiellement, le Cours, en particulier Iorsqu'il est comparé au Pan tendre, révèle combien I'innovation en milieu scolaire est autant que possible déproblématisée. En effet, un des objectifs de la scolarisation est l'acquisition du savoir et du pouvoir, et non pas de s'ouvrir à la pensée contestataire. Il est donc peu probable de trouver dans le Cours les épanchements véhéments que contiennent les souvenirs de Calelhon.

On peut alors mieux apprécier son flou rhétorique. Selon les passages, les exemples choisis et les périodes discutées, l'auteure soumet des interprétations souvent contradictoires. Ainsi, la culture populaire est tour à tour louée et critiquée. L'imprécision la plus notable concerne la définition de ce que Calelhon entend par «populaire »:

Tandis que le latin classique était en usage dans les écoles et parmi les classes lettrées, dans le peuple se répandit plus vite le latin vulgaire, le latin de la conversation, celui des soldats et des colons. De ce latin populaire... sortit peu à peu la langue romane, comprenant plusieurs dialectes, [dont] le dialecte d'oc.... La langue d'oc n'est donc pas un patois ; elle est une vraie langue ayant pour elle la noblesse de ses origines. (5)

Sans plus amples éclaircissements, le vocabulaire utilisé qualifie l'occitan de telle sorte à ne plus pouvoir le décrire, cette langue étant à la fois populaire et élitiste. Cette confusion l'emporte dans le Cours. Ainsi, Calelhon déplore la « décadence » de sa langue, qui, au fil des siècles, « se réfugia chez les classes populaires et de reine devint servante »(9). A plusieurs reprises, elle critique les textes occitans qui ne respectent pas une graphie normative et qui incluent de nombreux gallicismes. Néanmoins, Calelhon ne cesse d'exalter le résultat de cette « décadence », par exemple dans des jugements tels que celui-ci, lorsqu'elle observe que la littérature occitane peine à s'établir : La langue « fleurit toujours sur les lèvres des paysans et des gens de métiers.... Elle jaillit franche et drue de l'esprit de notre peuple, du cœur de notre terre » (9). La position développée est paradoxale : I'auteure se réjouit de pouvoir enfin enseigner sa langue comme langue littéraire et, pourtant, elle identifie dans la culture populaire, orale et sans prestige, la spécificité et l'intérêt de ladite langue. 
Une même ambiguïté se décèle dans le jugement que porte Calelhon sur la propagation du français. Commentant la prépondérance de la langue nationale au cours de l'histoire, Calelhon se plaint que « sa langue subissait l'empreinte tyrannique du français qui en faisait une espèce de métisse bâtarde et laideronne » (11). Pourtant, elle présente sa propre entreprise pédagogique comme un cours d'instruction civique et perçoit I'enseignement de la littérature occitane comme un élément concourant à la formation d'une unité nationale : «La physionomie de la France se dégage plus nette, plus vraie, plus diverse, variée, complexe, plus belle et plus émouvante dans son harmonieuse complexité » (16). Loin de s'émouvoir des droits bafoués de sa région, Calelhon est ici d'un avis modéré et les critiques tels que Anne-Marie Thiesse, pour qui la littérature régionale n'a guère cherché à remettre en cause les structures existantes, émettent une opinion qui s'applique parfaitement au Cours de Calelhon.

Indiscutablement, l'auteure du Cours est convaincue que la production de littérature, bien plus que des aspects socioculturels, atteste la vivacité de la langue. Si la tradition orale ne saurait être négligée, la tradition écrite (et félibréenne) est signe de raffinement et de réussite : Mistral « nous a révélé le miracle de la poésie et de l'art qui, partant de la réalité savamment observée, arrivent à s'élever jusqu'aux plus hauts sommets de la beauté éternelle » (51). Délaissant les conditions socioéconomiques longuement discutées dans Lo Pan tendre, Calelhon approche l'enseignement de la littérature occitane d'un point de vue limité à des considérations dépolitisées. Certes, elle n'oublie pas de mentionner les faits historiques qui ont conduit à la déchéance de la langue. Toutefois, son discours tient surtout à démontrer des idées d'une portée plus générale que les circonstances historiques ne le font, à savoir (du point de vue de Calelhon) que l'âme populaire trouve sa meilleure expression dans la haute littérature, que la littérature recèle la richesse de la langue et que toute langue mérite de produire des textes littéraires. Plus que les revendications sociales, ce qui compte est la recherche du beau idéal, de la forme et du style les plus agréables.

De telles remarques renvoient à la notion de sincérité, à laquelle Calelhon donne une importance décisive dans Lo Pan tendre. C'est, en effet, la sincérité de Mirèio, du moins telle que Calelhon la perçoit, qui I'a séduite et, en fin de compte, a bouleversé sa vie. C'est en découvrant l'œuvre de Mistral qu'elle décide de se lancer elle-même dans les lettres occitanes. Néanmoins, la sincérité manifestée dans Lo Pan tendre est, en partie en raison du genre et du public visé, d'une nature bien différente de la sincérité discutée dans le Cours. Dans celui-ci, la sincérité que Calelhon propose en modèle, ne consiste nullement en une identification subjective de la lectrice avec divers héros. Elle correspond plutôt à une représentation convaincante de la réalité. La sincérité n'est plus une émotion intense ; elle est, pour les écoliers, la reproduction littéraire, c'est-àdire modifiée, d'un vécu uniquement local observé par un habile spectateur. Dans le Cours, la sincérité est un savoir-faire dans la description régionaliste. Le traitement dans cet ouvrage de la carrière de Mistral est exemplaire. Ainsi, tout ce qui touche à son œuvre et à sa biographie semble sorti d'un livre aux visées grandioses portant précisément sur les humbles détails de la vie. Les écoliers penchés sur ces pages apprendront que I'histoire de Mistral est I'histoire de l'écriture née d'un vécu sans histoire et sans écrit. Calelhon rappelle que Lamartine disait de lui qu'il était le « Homère champêtre » de la Provence. La référence livresque est poussée jusqu'à la suggestion biblique : les parents de Mistral ne font-ils pas « songer à Ruth et 
Booz » (23-27) ? Toujours prête à citer Lamartine, Calelhon reprend le jugement de ce dernier : « Mireille, c'est la transfiguration de la nature et du cœur humain en poésie dans toute cette partie de la Basse-Provence » (27).

Ou dans une autre citation de Lamartine :

Voilà de ces épopées sur lesquelles les imaginations du peuple se façonnent, se polissent et font passer avec des récits enchanteurs de l'aïeul à l'enfant, de la mère à la fille, du fiancé à l'amante, toutes les bontés de l'âme, toutes les beautés de la pensée, toutes les saintetés de tous les amours qui font un sanctuaire du foyer du pauvre. (28)

On est loin de la sincérité de l'autobiographie de Calelhon. La sincérité du Cours désigne une éclatante transformation du populaire à l'idéal et de l'oral à l'écrit qui découle précisément d'une observation précise du milieu décrit. Il n'est plus simplement question de s'identifier avec toute la force des émotions, mais plutôt de déployer des techniques littéraires qui communiquent efficacement l'information voulue. La sincérité est une pratique littéraire nécessitant une parfaite maîtrise, de sorte que la recréation de l'expérience s'accompagne de son embellissement. La sincérité artistique est à la fois copie et perfectionnement. Alors que le Cours élimine toute revendication franchement sociale, la valorisation constante de la littérature et l'équation de la sincérité et du savoir-faire suggèrent qu'en dépit de toute prétention populaire, il n'y a qu'un but méritoire dans l'éducation : l'érudition.

Ce faisant, le manuel que rédige Calelhon est, comme le montre la citation suivante, un produit éminemment félibréen :

Le Félibrige combine astucieusement rupture et tradition. A l'invocation souvent floue de la richesse des siècles passés s'oppose.... la certitude d'être les premiers à donner à la langue sa véritable valeur. (Pasquini, « Félibrige », 259)

En fait, la langue félibréenne, bien qu'elle se veuille langue du peuple, est le fruit d'une «tradition inventée » (280). Elle « vit dans les nombreux discours dont le premier but est d'affirmer qu'elle est encore vivante », par exemple dans le discours littéraire, bien plus que dans une pratique quotidienne et véritablement populaire (Pasquini, Pays, 145-146). Par conséquent, le peuple devient « objet de discours »; entre autres, «il est prié... d'abandonner ses parlers dégradés et dégradants pour la dignité de la langue »(135). Le Cours de Calelhon s'intègre pleinement à cette logique. Présentée de telle manière, la littérature est la clé de toutes les réussites. La conclusion du Cours est révélatrice à cet égard. D’une part, Calelhon entrevoit la possibilité de l'extinction de I'occitan, mais, dit-elle, la renaissance littéraire de l'occitan inversera la tendance, car, grâce à elle, « la langue ne meurt pas » (69). D’autre part, on apprend que la littérature procure le réconfort en toutes circonstances, et Calelhon exhorte ses lecteurs à cultiver leur goût pour les belles-lettres : « Vous y trouveriez pour votre profit intellectuel et moral des joies certaines de l'esprit et du cœur »(69). On ne doit d'ailleurs pas s'étonner que Calelhon attache une grande importance à la sincérité des œuvres qu'elle décrit et, dans ses mémoires, à I'authenticité qu'elle perçoit dans l'héroïne de Mirèio. En effet, la question de la sincérité se pose de façon accrue dans le Félibrige. Nombreux sont ceux qui ont reproché aux Félibres «l'artificialité » de leur langue (Pasquini, «Félibrige », 267) ainsi que «l'illusion» souvent «sublimée » de vouloir recréer une culture disparue (282-283). Répondant implicitement à de telles vues, Calelhon proclame inlassablement l'authenticité de l'entreprise littéraire occitane et de sa propre démarche dans l'engagement félibréen. 
S'il ne se démarque pas de l'idéologie félibréenne, le travail de Calelhon ne se distancie pas non plus des pratiques établies dans l'histoire littéraire. Une des histoires littéraires de plus grand renom en France est le volume de Madame de Staël, De la Littérature considérée dans ses rapports avec les institutions sociales, datant de 1800, qui déjà conçoit un lien solide entre l'érudition et la morale grâce à l'étude des belles-lettres (20-26). Calelhon souscrirait sans doute également aux remarques émises dans Les Fondements de l'histoire littéraire, étude des principes régissant cette discipline, par exemple que «I'histoire littéraire se définit comme I'histoire morale d'une époque, comme l'étude du mouvement de la pensée, comme une approche de l'esprit d'un temps » (321), ou encore que I'histoire littéraire est simultanément travail d'érudition, avec la collection de sources importantes et une analyse de vaste portée, et de vulgarisation, puisqu'elle indique aux lecteurs une liste d'auteurs réputés tout en leur fournissant une interprétation générale de I'histoire des idées (388). Le travail de Calelhon est, de même, symptomatique des conditions historiques traditionnellement favorables à de semblables projets :

L'histoire littéraire est une création propre au XIXe siècle, bien qu'elle puisse se trouver des modèles dans un passé plus lointain : elle résulte d'une récente rupture avec le passé (due à la Révolution) et, après un retour au calme, d'une volonté réactionnelle, chez I'homme contemporain, de se situer à présent dans l'histoire universelle. (8)

Dans le contexte occitan, comment ne pas penser à la rupture que constitue l'abandon rapide de l'occitan au bénéfice du français dans la première moitié du vingtième siècle et aux souhaits de Calelhon et des Félibres de voir leur langue reconnue au-delà de ses frontières géographiques ainsi qu'en dehors des milieux populaires ? A ce propos, Calelhon conclut de l'attachement de Mistral à son terroir et de son œuvre occitane qu'ils font de lui « un grand poète universel»(26). Une fois devenue littéraire, la langue est promise au meilleur avenir : « Notre belle langue est un instrument assez souple, assez harmonieux, assez puissant, pour être capable d'interpréter toute la pensée humaine » (69). Calelhon prédit que «le secret de l'an qui vient » peut se résumer de la sorte : grâce à «l'harmonieuse diversité de ses provinces», le Midi montrera à tous que «la poésie est éternelle » (70). N'oublions pas néanmoins de placer le Cours dans son contexte immédiat. «L'an qui vient » est 1945 (le Cours paraît en 1944). Ecrit en pleine guerre, l'ouvrage élimine effectivement l'histoire contemporaine de ce qui est justement une histoire de la littérature. L'optimisme de Calelhon se veut probablement rassurant, mais dans une Europe en guerre, jusqu'à quel point peut-on rendre crédible l'affirmation qu'un « régionalisme intelligent», dans lequel l'occitan pourrait s'épanouir, est sur le point de naître (69) ? Comment une histoire littéraire, rédigée en période de violents bouleversements, peut-elle passer ceux-ci sous silence? Cette discrétion suffit à montrer combien le projet pédagogique du Cours se distancie de la réalité et ne trouve d'application que dans le lieu privilégié de l'environnement scolaire.

Bien que les langues minoritaires aient besoin d'histoire, car celle-ci leur est souvent refusée, le Cours prône, en fin de compte, une esthétique, et non une politique. En outre, il faut bien admettre qu'un pareil ouvrage n'a guère d'impact dans le milieu social dont il fait l'éloge. Comme le montre cruellement le cas occitan, étudier une langue dans un cours de littérature ne suffit pas à en garantir une pratique sociale florissante. Reprenant l'expression de Barthes, selon laquelle l'écriture est « un interdit de parole » (1322), on peut sans doute proposer une nouvelle formulation à la lecture du Cours, dans lequel l'écriture est un interdit de réalité, 
une mise à l'écart d'éléments qui, non littéraires, touchent pourtant au plus près le sort de la langue. Dans le Cours, la littérature et son étude demeurent une mise en fiction de l'expérience. Ce n'est sans doute pas un hasard si, pour combler ce manque d'attaches au réel, le Cours de Calelhon développe un discours savamment articulé sur le besoin de sincérité et d'authenticité.

\section{Ouvrages cités}

Barthes, Roland. «Réponses. » Fuvres complètes, tome 2. Paris : Seuil, 1994. 1307-24.

Calelhon. Cours de littérature occitane. Villefranche-de-Rouergue : Salingardes, 1944.

---. Lo Pan tendre : Al païs negre. Rodez : Grelh Roergàs, 1977.

Camproux, Charles. Histoire de la littérature occitane. Paris : Payot, 1953.

Fourié, Jean. « Regards toulousains, I'amitié avec Joseph Salvat. » Collòqui Calelhon - Eugène Séguret. Rodez : Grelh Roergàs, 1995. 127-40.

Fraisse, Luc. Les Fondements de l'histoire littéraire : de Saint-René Taillandier à Lanson. Paris : Champion, 2002.

Lafont, Robert. Quarante Ans de sociolinguistique à la périphérie. Paris : Harmattan, 1997.

Lafont, Robert, et Christian Anatole. Nouvelle Histoire de la littérature occitane. Paris : PUF, 1971.

Mistral, Frédéric. Mirèio. Avignon : Roumanille, 1859.

---. Memòri e raconte. Aix-en-Provence : Berenguié, 1969.

Pasquini, Pierre. Les Pays des parlers perdus. Montpellier : Languedoc, 1994.

---. « Le Félibrige : les formes d'une institution. » Dix Siècles d'usage et d'images de l'occitan : des troubadours à l'internet. Dir. Henri Boyer et Philippe Gardy. Paris : Harmattan, 2001. 257-89.

Séguret, Pierre. «Calelhon militante et le Journal de l'Aveyron. » Collòqui Calelhon - Eugène Séguret. Rodez : Grelh Roergàs, 1995. 99-106.

Staël, Madame de. De la Littérature considérée dans ses rapports avec les institutions sociales. Genève/Paris : Droz/Minard, 1959.

Sturm, Ernest. «René Wellek et la crise des idées littéraires. » Une Théorie de la critique moderne : la critique française, italienne et espagnole (1900-1950). By René Wellek. Paris : Corti, 1996. 7-24.

Thiesse, Anne-Marie. Ecrire la France : le mouvement littéraire régionaliste de langue française entre la Belle Epoque et la Libération. Paris : PUF, 1991. 